Technological University Dublin ARROW@TU Dublin

2014-09-23

\title{
Breast Cancer Detection Using Interferometric MUSIC: Experimental and Numerical Assessment
}

\author{
Giuseppe Ruvio \\ Technological University Dublin, Giuseppe.Ruvio@tudublin.ie \\ Raffaele Solimene \\ Seconda Università di Napoli \\ Antonio Cuccaro \\ Seconda Università di Napoli
}

See next page for additional authors

Follow this and additional works at: https://arrow.tudublin.ie/ahfrcart

Part of the Biomedical Engineering and Bioengineering Commons, and the Systems and

Communications Commons

\section{Recommended Citation}

G. Ruvio, R. Solimene, A. Cuccaro, D. Gaetano, J. E. Browne, and M. J. Ammann, "Breast cancer detection using interferometric MUSIC: experimental and numerical assessment", Medical Physics, vol. 41, issue 10, pp. 103101/1-11, 09/2014. doi:10.1118/1.4892067

This Article is brought to you for free and open access by the Antenna \& High Frequency Research Centre at ARROW@TU Dublin. It has been accepted for inclusion in Articles by an authorized administrator of ARROW@TU Dublin. For more information, please contact arrow.admin@tudublin.ie, aisling.coyne@tudublin.ie, gerard.connolly@tudublin.ie.

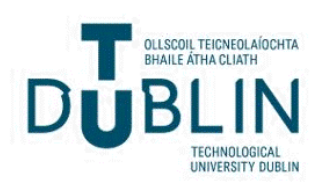


Authors

Giuseppe Ruvio, Raffaele Solimene, Antonio Cuccaro, Domenico Gaetano, Jacinta Browne, and Max Ammann

This article is available at ARROW@TU Dublin: https://arrow.tudublin.ie/ahfrcart/55 


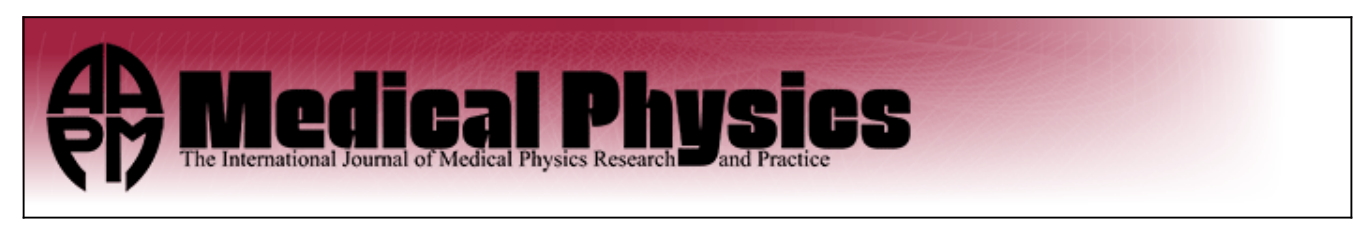

\section{Breast cancer detection using interferometric MUSIC: Experimental and numerical assessment}

Giuseppe Ruvio, Raffaele Solimene, Antonio Cuccaro, Domenico Gaetano, Jacinta E. Browne, and Max J. Ammann

Citation: Medical Physics 41, 103101 (2014); doi: 10.1118/1.4892067

View online: http://dx.doi.org/10.1118/1.4892067

View Table of Contents: http://scitation.aip.org/content/aapm/journal/medphys/41/10?ver=pdfcov

Published by the American Association of Physicists in Medicine

\section{Articles you may be interested in}

Integration of microwave tomography with magnetic resonance for improved breast imaging

Med. Phys. 40, 103101 (2013); 10.1118/1.4820361

Implementation and evaluation of an expectation maximization reconstruction algorithm for gamma emission breast tomosynthesis

Med. Phys. 39, 7580 (2012); 10.1118/1.4764480

Near-infrared spectral tomography integrated with digital breast tomosynthesis: Effects of tissue scattering on optical data acquisition design

Med. Phys. 39, 4579 (2012); 10.1118/1.4728228

Design and evaluation of a hybrid photoacoustic tomography and diffuse optical tomography system for breast cancer detection

Med. Phys. 39, 2584 (2012); 10.1118/1.3703598

Mechanically assisted 3D ultrasound guided prostate biopsy system

Med. Phys. 35, 5397 (2008); 10.1118/1.3002415

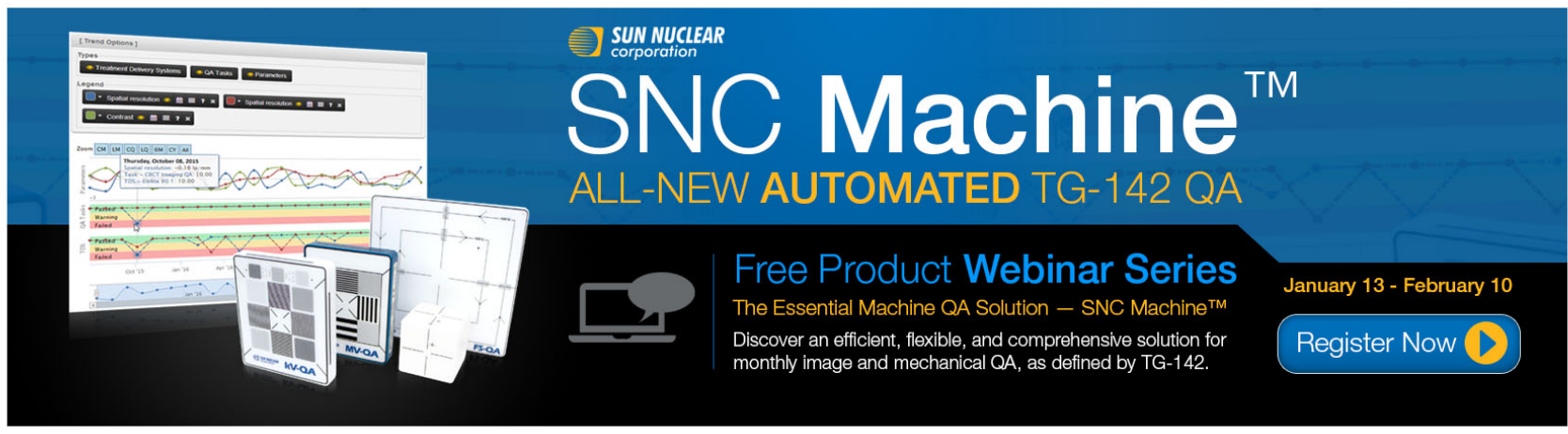




\title{
Breast cancer detection using interferometric MUSIC: Experimental and numerical assessment
}

\author{
Giuseppe Ruvio ${ }^{a)}$ \\ Antenna $\mathcal{E}$ Frequency Research Centre, Dublin Institute of Technology, Kevin Street, Dublin 8, 81031, Ireland \\ and Department of Industrial and Information Engineering, Seconda Università di Napoli, via Roma 56, \\ Aversa 81031, Italy
}

Raffaele Solimene and Antonio Cuccaro

Department of Industrial and Information Engineering, Seconda Università di Napoli, via Roma 56, Aversa 81031, Italy

Domenico Gaetano

AHFR Centre, Dublin Institute of Technology, Kevin Street, Dublin 8, 81031, Ireland

Jacinta E. Browne

School of Physics, Dublin Institute of Technology, Kevin Street, Dublin 8, 81031, Ireland

Max J. Ammann

AHFR Centre, Dublin Institute of Technology, Kevin Street, Dublin 8, 81031, Ireland

(Received 22 January 2014; revised 10 July 2014; accepted for publication 14 July 2014; published 23 September 2014)

Purpose: In microwave breast cancer detection, it is often beneficial to arrange sensors in close proximity to the breast. The resultant coupling generally changes the antenna response. As an a priori characterization of the radio frequency system becomes difficult, this can lead to severe degradation of the detection efficacy. The purpose of this paper is to demonstrate the advantages of adopting an interferometric multiple signal classification (I-MUSIC) approach due to its limited dependence from a priori information on the antenna. The performance of I-MUSIC detection was measured in terms of signal-to-clutter ratio (SCR), signal-to-mean ratio (SMR), and spatial displacement (SD) and compared to other common linear noncoherent imaging methods, such as migration and the standard wideband MUSIC (WB-MUSIC) which also works when the antenna is not accounted for.

Methods: The data were acquired by scanning a synthetic oil-in-gelatin phantom that mimics the dielectric properties of breast tissues across the spectrum $1-3 \mathrm{GHz}$ using a proprietary breast microwave multi-monostatic radar system. The phantom is a multilayer structure that includes skin, adipose, fibroconnective, fibroglandular, and tumor tissue with an adipose component accounting for $60 \%$ of the whole structure. The detected tumor has a diameter of $5 \mathrm{~mm}$ and is inserted inside a fibroglandular region with a permittivity contrast $\varepsilon_{r \text {-tumor }} / \varepsilon_{r \text {-fibroglandular }}<1.5$ over the operating band. Three datasets were recorded corresponding to three antennas with different coupling mechanisms. This was done to assess the independence of the I-MUSIC method from antenna characterizations. The datasets were processed by using I-MUSIC, noncoherent migration, and wideband MUSIC under equivalent conditions (i.e., operative bandwidth, frequency samples, and scanning positions). SCR, SMR, and SD figures were measured from all reconstructed images. In order to benchmark experimental results, numerical simulations of equivalent scenarios were carried out by using CST Microwave Studio. The three numerical datasets were then processed following the same procedure that was designed for the experimental case.

Results: Detection results are presented for both experimental and numerical phantoms, and higher performance of the I-MUSIC method in comparison with the WB-MUSIC and noncoherent migration is achieved. This finding is confirmed for the three different antennas in this study. Although a delocalization effect occurs, experimental datasets show that the signal-to-clutter ratio and the signal-to-mean performance with the I-MUSIC are at least 5 and 2.3 times better than the other methods, respectively. The numerical datasets calculated on an equivalent phantom for cross-testing confirm the improved performance of the I-MUSIC in terms of SCR and SMR. In numerical simulations, the delocalization effect is dramatically reduced up to an SD value of 1.61 achieved with the I-MUSIC in combination with the antipodal Vivaldi antenna. This shows that mechanical uncertainties are the main reason for the delocalization effect in the measurements.

Conclusions: Experimental results show that the I-MUSIC generates images with signal-to-clutter levels higher than $5.46 \mathrm{~dB}$ across all working conditions and it reaches $7.84 \mathrm{~dB}$ in combination with the antipodal Vivaldi antenna. Numerical simulations confirm this trend and due to ideal mechanical conditions return a signal-to-clutter level higher than $7.61 \mathrm{~dB}$. The I-MUSIC largely outperforms the 
methods under comparison and is able to detect a 5-mm tumor with a permittivity contrast of 1.5. (C) 2014 American Association of Physicists in Medicine. [http://dx.doi.org/10.1118/1.4892067]

Key words: electromagnetic inverse scattering, breast cancer detection, microwave imaging

\section{INTRODUCTION}

Breast cancer is the most common type in women and incidences are increasing in the developing world due to extended life expectancy, increased urbanization, and expansion of Western lifestyles. The World Health Organization launched a key message to stress the importance of "early detection in order to improve breast cancer outcome and survival remains the cornerstone of breast cancer control."1 So far the only breast cancer screening method that has proved to be effective is mammography screening. However, its detection capabilities have been shown to be limited due to poor benign/malignant tissue contrast (around 10\%). Between $4 \%$ and 34\% of all breast cancers are missed and nearly $70 \%$ of all breast lesions turn out to be benign. ${ }^{2}$ The exposure to low levels of ionizing radiation may reduce patient compliance with screening. On the other hand, magnetic resonance imaging (MRI) offers higher performance in terms of resolution and, hence, a correct diagnosis. Typical resolution in a breast image obtained via a 3 tesla (3 T) MRI system is about $1 \mathrm{~mm}$. However, MRI is still expensive and not proven to be a practical procedure for wide screening campaigns. Ultrasound can supplement x-ray mammograms by discerning between liquid cysts and solid tumors. Alternative methods at exploratory stage are based on different tissue parameters such as elasticity, temperature, and optical properties. Exploiting dielectric contrast to image relevant features in biological targets has been investigated for decades ${ }^{3}$ but recently this research area has gained critical mass. Higher spatial resolution is achievable by using $\mathrm{x}$-ray radiation, but radio frequency (RF) technology offers higher dielectric contrast between normal and diseased breast tissues which can supplement conventional diagnostics. This can enable the reduction of false diagnosis with consequent high social impact and reduced costs to health systems. Moreover, it is based on low power nonionizing radiation which facilitates cost-competitive screenings. These important potential outcomes have triggered the investigation of many microwave imaging techniques, aimed at detecting, localizing, and identifying tumors in breast tissues. ${ }^{4}$ However, while the contrast between malignant and adipose breast tissues may be as large as 10 , those between malignant and healthy fibroglandular tissues can be as low as $10 \%$, in both permittivity and conductivity. 5,6 This places an important challenge in microwave breast imaging as most breast tumors appear in the fibroglandular tissues.

Several microwave imaging techniques were proposed in the last two decades. These can be generally grouped into nonlinear and linear inversions. Nonlinear inversions aim at retrieving the breast dielectric and conductivity profiles. ${ }^{7,8}$ However, they are computationally intensive ${ }^{9}$ and can suffer from convergence and reliability problems due to false solutions. ${ }^{10}$ Linear methods (also addressed as radar focusing) are robust and computationally effective but they only allow detection and localization of the tumor inhomogeneities ${ }^{11}$ with the requirement for further classification steps. ${ }^{12}$ In any case, the corresponding inverse scattering problem is very difficult as the tumor is buried within a highly inhomogeneous medium.

Imaging algorithms are only part of the picture. A crucial role is played by the RF system and in particular by the antennas. Ultra-wideband (UWB) antennas have been proposed for this application as they can offer a very large operating band, stable radiation properties, and compact dimensions both with planar ${ }^{13-15}$ and 3D profiles. ${ }^{16}$ In order to fully assess the achievable performance of a breast cancer detection system, the presence of the antenna must be taken into account. Ideal sources are worthy of consideration at a preliminary stage when the focus is limited to the algorithm. Recent contributions deal with more complex scenarios where antennas are considered in the numerical model. ${ }^{17,18}$ Also a number of antenna prototypes were developed and integrated into experimental phantom-based systems, ${ }^{19}$ under preclinical ${ }^{20}$ and clinical trials. ${ }^{21,22}$

Accurate near-field microwave imaging requires characterization/equalization of the antenna behavior. This can be pursued by a suitable set of measurements or numerical simulations. However, as the breast properties change from patient to patient, residual errors still remain. With uncertainty levels as high as the magnitude of the tumor scattered field, the imaging procedure robustness is dramatically endangered. In particular, dense breasts are particularly exposed to this problem as they present lower tumor/healthy-tissue contrast.

In order to mitigate the requirement for antenna characterization in complex near-field scenarios, suitable detection methods should be properly devised. Interferometric multiple signal classification (I-MUSIC) was developed ${ }^{17}$ to address this issue. This approach represents a multifrequency variant of the well-known time-reversal MUSIC (Ref. 23) when adapted to a multi-monostatic configuration. The term interferometric refers to the diversity created by using different frequency samples. More specifically, images obtained at different frequencies are multiplicatively mixed with each other. Theoretical details and performance assessment were addressed by Solimene et al. ${ }^{24}$ Preliminary numerical results on a breast scenario were reported by Ruvio et al. ${ }^{25}$ In this study, a 2D numerical mostly fatty breast model was derived from a MRI scan according to the Wisconsin's repository. ${ }^{26}$ It is shown that the method succeeds in detecting a low-contrast tumor when the antenna response is completely neglected in the imaging procedure. As the scattered signal coming from the tumor is generally weaker than internal reflections in the antenna and reflections coming from the skin layer and other benign tissues, clutter removal procedures are an important step in the diagnostic system. Several clutter-rejection methods have been proposed in the literature. Some of them rely on 
filtering procedures that attempt to estimate the clutter signal at a given position and subtract it from the actual measurements. ${ }^{27}$ Alternatively, some procedures employ a differential scheme where two sets of measurements corresponding to two different rotations of the system are subtracted from each other. ${ }^{28}$ These procedures strongly depend on the uniformity of the skin layer, breast shape, and antenna response. Moreover, they can also result in filtering part of the signal weakly scattered by the tumor scattered signal. Time gating procedures can exploit entropic metrics that enables identification of time windows in which signals have to be silenced ${ }^{29}$ but rely on similar assumption about the breast.

Subspace projection methods also entail filtering the scattered signals but being based on amplitude differences do not require uniformity assumptions. Sarafianou $e t$ al. proposed a novel skin reflection removal algorithm ${ }^{30}$ that operates starting from a preliminary breast surface estimation which is then used to create a synthetic phantom. Finally, the field scattered by this synthetic phantom is subtracted from the actual measurements.

Preclinical testing requires the realization of accurate experimental phantoms with varying degrees of complexity. ${ }^{31}$ In general, the realization of synthetic materials that closely mimic the physical properties of various human tissues becomes very difficult when a large operating frequency range is required.

In this paper, the achievable performance of the I-MUSIC in combination with a subspace-based anticlutter technique is experimentally evaluated with a $2 \mathrm{D}$ inhomogeneous breast phantom that was manufactured with oil-in-gelatin emulsions. These tissue-mimicking materials are able to reproduce the electrical properties of different normal and malignant breast tissues. ${ }^{32}$ Different dielectric properties can be obtained by varying the percentages of a $50 \%$ kerosene- $50 \%$ safflower oil solution to simulate the electric behavior of healthy and diseased breast tissues. Experimental data are obtained by using three different types of antennas (i.e., a planar monopole, a semifolded monopole, and an antipodal Vivaldi antenna). Data are then processed to detect a 5-mm tumor included within a fibroglandular region. Moreover, experimental results were cross-referenced against equivalent numerical model simulations. The achievable performance obtained by I-MUSIC is then compared with two other methods in the literature: the noncoherent migration, ${ }^{33}$ which is a particular version of beamforming, and the wideband MUSIC (Ref. 34) which belongs to the class of spectral estimation algorithms as IMUSIC. These three methods under comparison are noncoherent in the sense that reconstructions are obtained only by noncoherent amplitude superimposition of images obtained at different frequencies. The aim of this paper is to highlight the enhanced detection performance achieved by using I-MUSIC when no a priori antenna characterization is given. To the best of our knowledge, this paper is the first to analyze and quantify, through experimental and numerical data, the detection capabilities of I-MUSIC by using three noncharacterized antennas with different antenna/tissues coupling mechanisms.

\section{PHANTOM AND MEASUREMENT SETUP}

Breast phantoms based on oil-in-gelatin emulsions can reproduce the electrical properties of various normal and malignant breast tissues. An essential property of these materials is the capability to create heterogeneous anthropomorphic structures with long-term stability of mechanical and electromagnetic properties. Moreover, due to their gelatinous consistence, these materials are convenient for relatively easy and inexpensive manufacturability as well as being characterized for the radio frequency range of interest in this study. For these reasons oil-in-gelatin materials were selected to manufacture a heterogeneous 2D phantom [Fig. 1(a)] including skin, adipose, fibroconnective, fibroglandular, and tumor tissues. Two dimensional phantoms (if not 1D) represent a convenient and common choice for a faster and fair system assessment. ${ }^{14,35-38}$ In particular, phantom simplification enables a more detailed investigation when nonideal antennas are used. ${ }^{18}$

Different tissues were realized by properly mixing the $50 \%$ kerosene-50\% safflower oil solution with a formaldehydebased emulsion. The following oil percentages were used to make the tissues: $80 \%$ oil concentration for the adipose tissue, $40 \%$ oil for fibroconnective tissue, $30 \%$ oil for fibroglandular and skin tissues, and $20 \%$ oil for the tumor. ${ }^{31}$ The phantom has an overall diameter of $114 \mathrm{~mm}$ including the 2 -mm-thick skin layer. The fibroconnective and fibroglandular regions have 68 and $20 \mathrm{~mm}$ diameters, respectively. The multilayer structure was realized with a multistage procedure: First, the adipose tissue was poured into a mold where a rod was introduced to make room for the fibroconnective/fibroglandular/tumor structure. After the adipose tissue had gelled, the same procedure was repeated for the other internal layers. Finally, a 2-mm-thick skin tissue was attached to the adipose tissue. Based on such adipose composition, this breast model can be categorized into heterogeneous mix (31\%-84\% adipose). ${ }^{6}$ In order to create a challenging detection scenario, the 5-mm-diameter tumor was asymmetrically located inside the fibrograndular region.

The dispersive behavior of skin, adipose, fibroconnective, fibroglandular, and tumor properties was measured over a large frequency range spanning from 1 to $3 \mathrm{GHz}$ using a coaxial probe [Fig. 1(b)]. As can be seen, the real part of the dielectric permittivities is reasonably close to the average values measured by Lazebnik et al. ${ }^{6}$ The tissues realized for this study present a conductivity contrast between benign and tumor tissues which is lower than the average values measured by Lazebnik et al. ${ }^{6}$ In particular, the overall permittivity between the tumor and the fibrograndular tissue is no greater than 1.5:1, which can be considered relatively low. The phantom was also imaged using MRI in order to assess its compliance from a morphologic perspective as well. The MR images were acquired on a $3 \mathrm{~T}$ system (Achieva, Philips, the Netherlands) using a high resolution $3 \mathrm{D} T_{2}$-weighted imaging sequence with a spatial resolution of $0.7 \times 0.7 \times 0.7 \mathrm{~mm}^{3}$. From Fig. 2(a) it can be appreciated that the desired layering was actually achieved.

The system antenna + phantom was immersed into a coupling medium which presents properties equivalent to the 


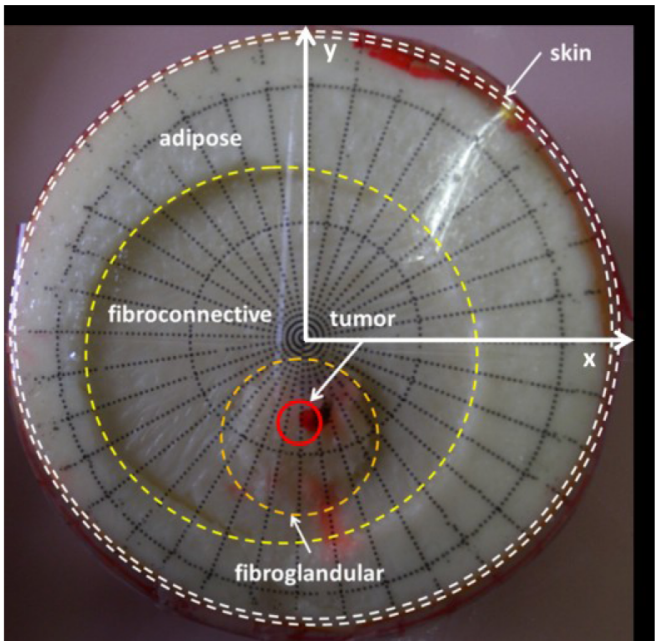

(a)

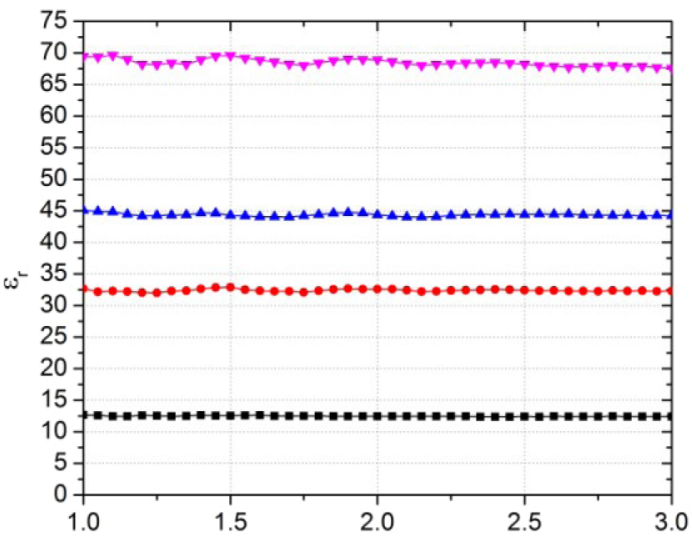

(b) Frequency $[\mathrm{GHz}]$

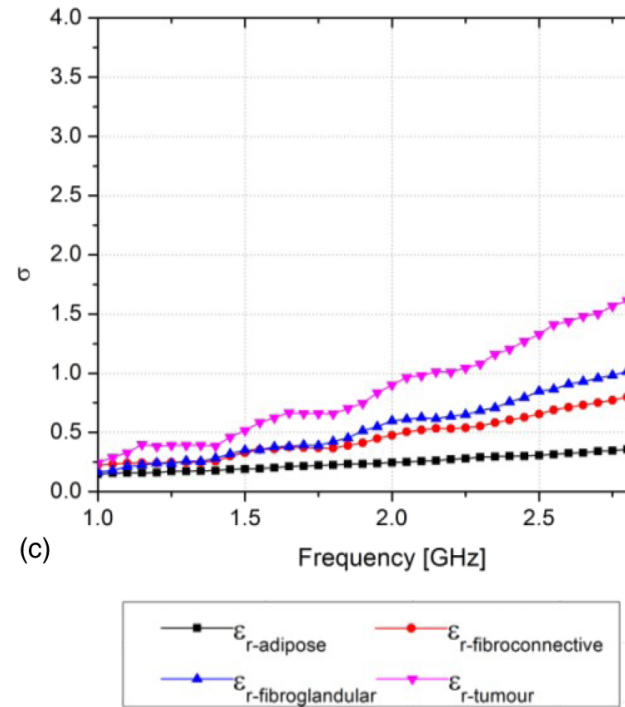

Fig. 1. (a) Cross-section of the cylindrical phantom, (b) measured relative permittivity of tissues, (c) measured conductivity of tissues.

expected adipose tissue. In particular, the average value of measured permittivity of the coupling medium and adipose tissue across the operating spectrum $1-3 \mathrm{GHz}$ is equal to 12. The adipose component is in fact dominant in the breast tissue distribution. The coupling medium was realized with

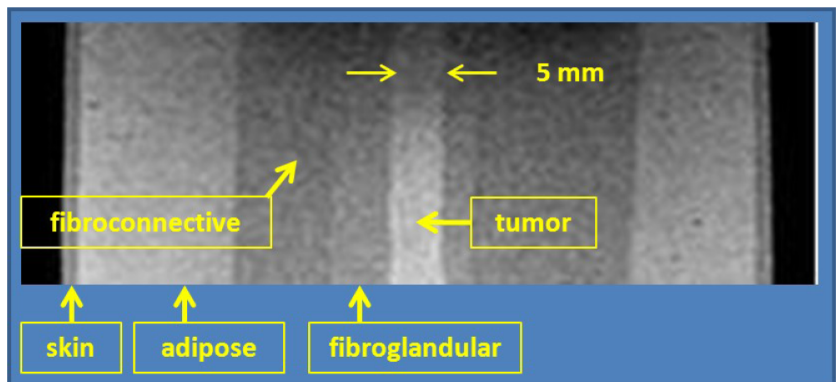

(a)

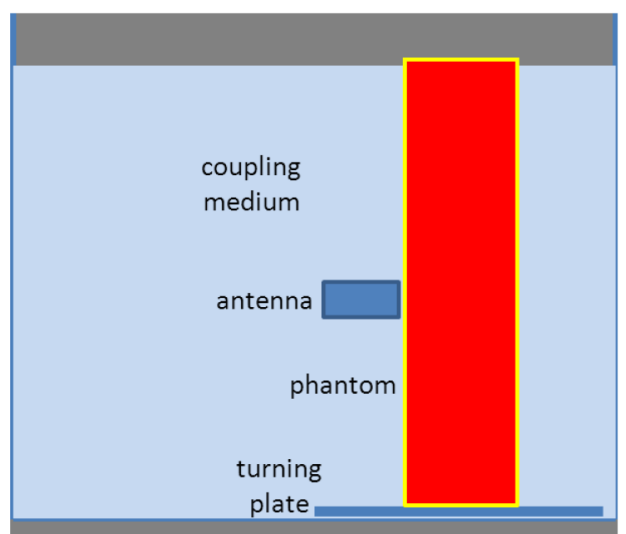

(b)

FIG. 2. (a) Slice through a 3D MRI phantom dataset, showing the internal layers; (b) the measurement setup showing the turntable-mounted phantom and antenna in the coupling medium.

the same $80 \%$ oil emulsion used to make the adipose tissue in the phantom but without gelling agents. The medium improves coupling from the antenna to the phantom and also enables antenna miniaturization. With the phantom placed on a turntable, frequency-domain scattering measurements were made over $360^{\circ}$ in $10^{\circ}$ steps [Fig. 2(b)]. Preliminary results based on measurements carried out with this phantom were presented with a planar monopole used as sensor. ${ }^{39}$

\section{THE ANTENNAS}

As antennas are generally characterized in terms of farfield behavior, the choice of best performing antenna for this particular imaging/sensing application is not obvious. On the one hand, monopole antennas present very stable behavior and are resilient to detuning when close to human tissue. On the other hand, properly oriented Vivaldi antennas provide greater coupling to adjacent human tissue. Figure 3 shows prototypes of the three antennas ${ }^{40}$ used for this comparative analysis. The three antennas were optimized to operate with a minimum $10-\mathrm{dB}$ return loss across the $100 \%$ bandwidth ranging from 1 to $3 \mathrm{GHz}$ when immersed in a coupling medium with $\varepsilon_{r}=12$. The dielectric substrate used in all three designs is 1.58 -mm-thick Taconic CER10 material. Figure 3(a) shows a printed planar monopole. The bottom edge of the radiating element was beveled to extend a good impedance match over a larger frequency range. The front view and a section of the semifolded monopole are depicted in Fig. 3(b). This antenna consists of a printed part and an 
(a)

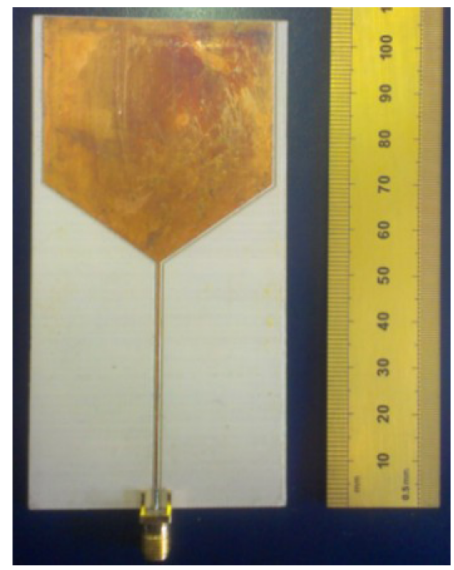

(b)

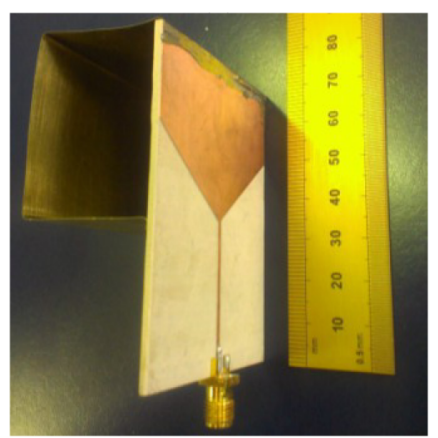

(c)

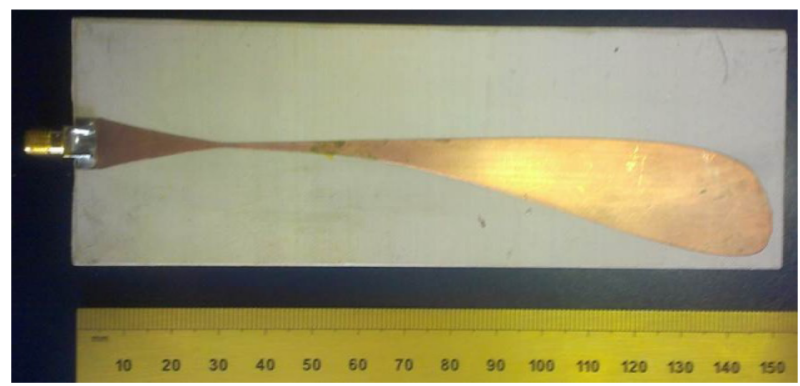

FIG. 3. Geometry of the antennas investigated: (a) planar monopole, (b) semifolded monopole, and (c) antipodal Vivaldi antenna.

external 0.2-mm-thick brass element. It is worth noticing that the front area of the planar monopole is reduced by $39 \%$ when the antenna is modified into a semifolded geometry. Finally, Fig. 3(c) shows the printed antipodal Vivaldi antenna. The tapering of the two arms is based on spline curves whose generating points were determined with an efficient global optimization (EGO) algorithm. The antennas under test were placed against the multilayer breast phantom so to have the printed radiating element lying on the $x z$-plane and centrally placed against the phantom according to the reference axis in Fig. 1(a). In particular, the Vivaldi antenna is positioned in such a way that the edge corresponding to the aperture of the arms and lying on the $x$ axis is centrally placed against the multilayer system. The planar monopole, the semifolded monopole, and the Vivaldi antenna present very different coupling/radiation mechanisms. The planar monopole tends to couple energy in an omnidirectional pattern in its azimuth plane, whereas the semifolded monopole can convey more power in one direction (toward the phantom). Finally, the
Vivaldi antenna combines good matching and directive stable coupling/radiation pattern across a large frequency range.

\section{THE IMAGING PROCEDURE}

The field scattered by the phantom is collected by a TX/RX antenna over $N$ scanning positions $\left(r_{o 1}, r_{o 2}, \ldots, r_{o N}\right)$ taken uniformly around it at $N_{f}$ frequency bins. The data of a complete scan can be accordingly arranged in the $N \times N_{f}$ scattering matrix $\mathbf{S}=\left[S^{1} \cdots S^{n} f \cdots S^{N_{f}}\right]$, where $S^{n}$ is the column vector of data collected over the observation positions at $n_{f}$-th frequency. These data include the tumor signal but also strong clutter components generated from the antenna's internal reflection, skin, and other benign tissues. As the clutter tends to mask the tumor signal, it has to be reduced before the image construction procedure.

A clutter-rejection subspace-based technique is adopted here. ${ }^{41}$ This method is chosen for not relying on unrealistic breast uniformity assumption nor on strong correlation between different time traces. Only the a priori assumption that clutter magnitude is higher than tumor signals is exploited. To this end, it is convenient to express the scattering matrix $\mathbf{S}$ through its singular value decomposition (SVD) as $\mathbf{S}=\mathbf{U} \Lambda \mathbf{V}^{H}$, where $\mathbf{U}$ and $\mathbf{V}$ are unitary matrices containing the left and right singular values, respectively, $(\cdot)^{H}$ represents the Hermitian operator, and $\Lambda$ is a diagonal matrix containing the singular values $\lambda_{1}, \lambda_{2}, \ldots, \lambda_{P}$, in decreasing order, with $P=\min \left\{N_{f}, N\right\}$. Clutter can be then mitigated by disregarding the projection of the scattering matrix on the singular functions corresponding to the highest singular values. The number of projections to discard generally depends on the source of clutter and requires information which is not $a$ priori known. Therefore, for this application, it is chosen only to discard the projection of the scattering matrix over the singular function corresponding to the highest singular value. Due to reflections in the antenna and from the skin layer, it can be considered true that the first singular function mainly contains clutter contribution. This choice can be considered conservative but realistic at the same time. Consequently, the decluttered data matrix is obtained as

$$
\mathbf{S}_{d}=\sum_{k=2}^{P} \lambda_{k} \mathbf{u}^{k} \mathbf{v}^{k H}
$$

with $P \leq \min \left(N, N_{f}\right)$ and $\mathbf{u}^{k}$ and $\mathbf{v}^{k}$ being the $k$ th column vectors of $\mathbf{U}$ and $\mathbf{V}$, respectively. With the clutter removed, the scattering data vectors (i.e., the columns of $\mathbf{S}_{d}$ ) can be approximated at the $n_{f}$-th working frequency by

$$
S_{d}^{n_{f}}=H^{R}\left(f_{n_{f}}\right) \mathbf{A}\left(f_{n_{f}}\right) H^{T}\left(f_{n_{f}}\right) \mathbf{b}\left(f_{n_{f}}\right),
$$

where $H^{T}\left(f_{n_{f}}\right)$ and $H^{R}\left(f_{n_{f}}\right)$ are the TX/RX antenna responses, respectively, $\mathbf{b}\left(f_{n_{f}}\right)$ is the vector of the scattering coefficients, and $\mathbf{A}\left(f_{n_{f}}\right)$ is the propagator which links the scatterers [located at $\left(r_{1}, r_{2}, \ldots, r_{M}\right)$ ] to the scattered field data. In particular, $S_{d}^{n_{f}}$ is the $n_{f}$-th column of $\mathbf{S}_{d}$ (which corresponds to the $n_{f}$-th adopted frequency) and represents the field scattered by the targets (assumed to be pointlike) 
and collected over the observation spatial positions. Since a multi-monostatic configuration is considered, the $l$ th column of $\mathbf{A}\left(f_{n_{f}}\right)$ is expressed as

$$
A^{l}\left(r_{l} ; f_{n_{f}}\right)=\left[G^{2}\left(r_{o 1}, r_{l} ; f_{n_{f}}\right), \ldots, G^{2}\left(r_{o N}, r_{l} ; f_{n_{f}}\right)\right]^{T},
$$

where $G\left(. ; f_{n_{f}}\right)$ is the $2 \mathrm{D}$ Green's function relative to the breast host medium, $G\left(r_{o n}, r_{l} ; f_{n_{f}}\right)=H_{0}^{2}\left(k_{\mathrm{eq}}\left|r_{o n}-r_{l}\right|\right)$, with $k_{\mathrm{eq}}$ being the propagation constant relative to an equivalent breast permittivity $\varepsilon_{r \text {-equivalent }}$ assigned equal to $\varepsilon_{r \text {-coupling_medium. }}$. $A^{l}\left(r_{l} ; f_{n_{f}}\right)$ accounts for the squared Green's function (due to the round-trip path) and the antenna's TX/RX responses. Some remarks are now appropriate. First, it is noted that in Eq. (2) mutual scattering between different tumors was neglected. This is actually a reasonable assumption because the breast is a highly lossy medium. Second, a multi-monostatic configuration was adopted. This choice is justified for the necessity to develop an imaging method that does not rely on the antenna response. In a multi-monostatic configuration, $H^{T}\left(f_{n_{f}}\right)$ and $H^{R}\left(f_{n_{f}}\right)$ can be considered nearly constant across the different scanning positions. However, antenna responses, $H^{T}\left(f_{n_{f}}\right)$ and $H^{R}\left(f_{n_{f}}\right)$, depend on frequency as well. Hence, as they are actually considered unknown in Eq. (2), data at different frequencies cannot be coherently combined while forming the images. However, data can be separately processed at each single frequency and the corresponding reconstructions can be then suitably combined. To this end, the I-MUSIC is proposed. ${ }^{24}$ First, for each adopted frequency, the correlation matrix is obtained as

$$
\mathbf{R}\left(f_{n_{f}}\right)=S_{d}^{n_{f}} S_{d}^{n_{f} H} .
$$

Since multiple views are not exploited, such a matrix is rank deficient with rank equal to one. Thus the pseudospectrum can be conveniently built without singular spectrum computation as

$$
\Phi^{n_{f}}\left(r_{k} ; f_{n_{f}}\right)=1 /\left\|\mathbf{P}^{n_{f}} A_{k}\left(f_{n_{f}}\right)\right\|^{2},
$$

where $\mathbf{P}^{n_{f}}=\mathbf{I}-\mathbf{Q}^{n_{f}}=\mathbf{I}-\mathbf{R}\left(f_{n_{f}}\right) /\left\|S_{d}^{n_{f}}\left(f_{n_{f}}\right)\right\|^{2}$ is a projector onto the noise subspace and $A_{k}\left(f_{n_{f}}\right)=A^{k}\left(r_{k} ; f_{n_{f}}\right) /$ $\left\|A^{k}\left(r_{k} ; f_{n_{f}}\right)\right\|$ is the steering vector evaluated at the trial positions $r_{k}$ within the spatial domain $D$ under investigation. It is also remarked that, as no a priori information regarding the phantom is given, the steering vector is constructed by employing the Green's function of an equivalent homogeneous breast medium. This is done by assigning the dielectric permittivity of the coupling medium to the equivalent breast medium in the Green's function expression.

Rank deficiency of $\mathbf{R}\left(f_{n_{f}}\right)$ entails some limitations on the achievable performance and uniqueness problems, especially with a low number of scanning positions. ${ }^{24}$ In order to overcome these limitations, pseudospectra obtained at different frequencies can be incoherently combined as suggested by Yavuz and Teixeira ${ }^{34}$ By doing so, the so-called wideband MUSIC is obtained

$$
\Phi_{\text {WB-MUSIC }}\left(r_{k}\right)=1 / \sum_{n_{f}=1}^{N_{f}}\left\|\mathbf{P}^{n_{f}} A_{k}\left(f_{n_{f}}\right)\right\|^{2} .
$$

In this contribution, single frequency pseudospectra are combined in an interferometric arrangement as done by Ruvio et al. ${ }^{25}$ The multifrequency pseudospectrum is overall built as

$$
\Phi_{\text {I-MUSIC }}\left(r_{k}\right)=1 / \prod_{n_{f}=1}^{N_{f}}\left\|\mathbf{P}^{n_{f}} A_{k}\left(f_{n_{f}}\right)\right\|^{2} .
$$

Hence, it consists in multiplying pixel by pixel the pseudospectra obtained at different frequencies.

Both methods are herein compared. As beamforming methods are largely employed in this kind of application, it is interesting to consider also an algorithm taken from this class in the comparison. To this end, the following noncoherent migration $(\mathrm{N}-\mathrm{M})^{11}$ approach is also taken into account

$$
\Phi_{\mathrm{N}-\mathrm{M}}\left(r_{k}\right)=\sum_{n_{f}=1}^{N_{f}}\left\|\mathbf{Q}^{n_{f}} A_{k}\left(f_{n_{f}}\right)\right\|^{2} .
$$

In the Appendix it is shown that Eq. (8) actually represents a particular beamforming algorithm where the time windowing is not imposed. This corresponds to the fact that no information about the antenna is available. In particular, the travel time within the antenna, which is required to set the time integration window, is not known.

\section{DATA ACQUISITION AND RESULTS}

The phantom was scanned radially over $360^{\circ}$ with a $10^{\circ}$ angular step (36 scanning positions) according to a multimonostatic radar configuration. In all experiments, the reflections from the antenna $\left(S_{11}\right)$ in the frequency domain (range 1-3 GHz) were recorded using a Rohde \& Schwarz ZVB8 vector network analyzer, which operated both as the microwave signal generator and as the recording device. The antennas were positioned against the phantom by using a rigid coaxial cable and Perspex holders which are transparent in the adopted frequency interval. The phantom was scanned at room temperature with the three different antennas previously mentioned. Ten frequency samples $\left(N_{f}=10\right.$, according to previous notation) uniformly distributed in the frequency range $1-3 \mathrm{GHz}$ were adopted for the detection procedures. From the different recorded scans operated by using the three antennas under assessment, reconstructions were carried out with the three noncoherent methods under equivalent conditions (i.e., $N$ and $N_{f}$ ). The reconstructions corresponding to three different detection methods used to process the measurements data are shown in Fig. 4 for all antennas under consideration. Although the target results delocalized in all reconstructions, the I-MUSIC method offers a better performance when compared to the other noncoherent approaches for enabling more focus and better dynamic range. Together with visual reconstructions of the detection algorithms, their performance was also measured by suitable metrics such as signal-to-clutter ratio (SCR) within breast, signal-to-mean ratio (SMR), and spatial displacement (SD). The SCR within breast compares the maximum tumor response to the maximum clutter response in the same reconstructed image. The 

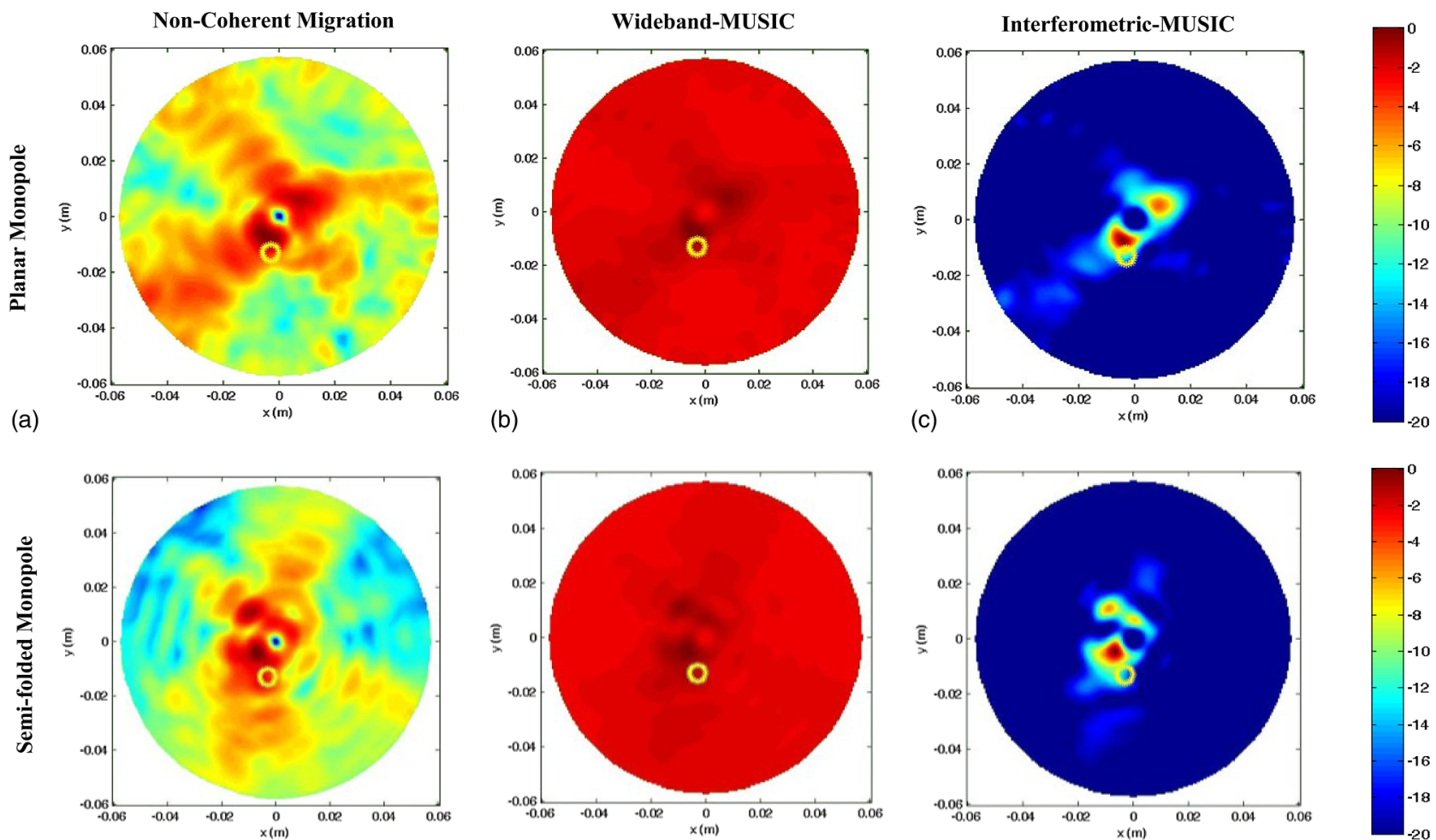

(e)

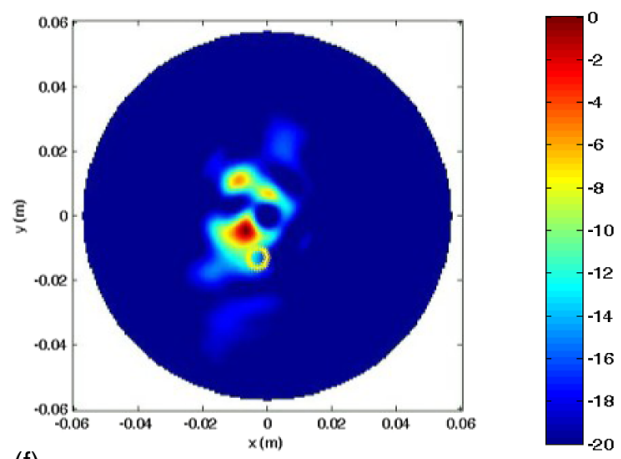

(d)

(f)
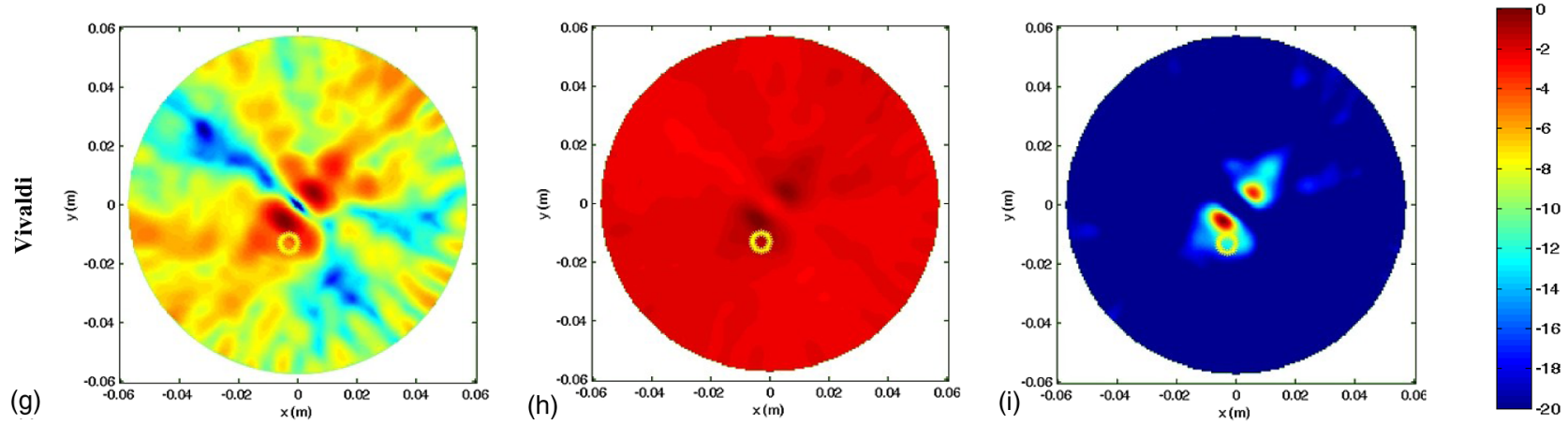

FIG. 4. Reconstructions based on experimental results. Each reconstruction is normalized to the corresponding maximum value and is expressed in decibel-scale. Tumor marked with yellow circle.

value of maximum clutter corresponds to the maximum pixel value of the image excluding the area which comprehends the tumor peak response up to twice the extent of the full width half maximum (FWHM) response of the tumor itself. The FWHM corresponds to the distance between the peak tumor response and the point at which the energy of the peak response drops by $3 \mathrm{~dB}$. The SMR compares the maximum tumor response in the reconstructed image with the average clutter response in the same image, whereas the SD measures the error in tumor localization and accounts for the difference between the tumor position as peak value in the reconstruction and as actual center position in the scanned phantom. Results are summarized in Table I and confirm the superior performance achieved by I-MUSIC in reconstructions. It is worth noting that the relatively high displacement expressed in terms of SD could be expected as a consequence of the approximated equivalent breast relative permittivity used in the steering vector formation [Eq. (5)]. For the experimental WB-MUSIC case, metrics could not be extracted as its dynamic range is smaller than the $3-\mathrm{dB}$ scale adopted for their calculation.

TABLE I. Reconstruction metrics. Experimental results.

\begin{tabular}{llrc}
\hline \hline Method & $\begin{array}{l}\text { SCR } \\
(\mathrm{dB})\end{array}$ & $\begin{array}{c}\text { SMR } \\
(\mathrm{dB})\end{array}$ & $\begin{array}{c}\text { SD } \\
(\mathrm{mm})\end{array}$ \\
\hline Coplanar monopole & & & \\
$\quad$ N-M & 1.13 & 8.53 & 27.2 \\
$\quad$ I-MUSIC & 5.87 & 20.30 & 26.6 \\
Semifolded monopole & & & \\
$\quad$ N-M & 0.89 & 9.16 & 24.7 \\
$\quad$ I-MUSIC & 5.46 & 21.52 & 25.1 \\
Vivaldi & & & \\
$\quad$ N-M & 1.49 & 9.78 & 26.3 \\
I-MUSIC & 7.84 & 26.48 & 26.9 \\
\hline \hline
\end{tabular}


Delocalization effects occur in the reconstructions from measured datasets due to the following issues in the mechanical setup:

- The phantom is rotated with a $10^{\circ}$ step but the tolerance of accuracy of the turntable is $\pm 2^{\circ}$.

- Although the antenna was held in place by using a Perspex frame, the interface between antenna and phantom was kept fixed at each scanning position with a tolerance of $\pm 1 \mathrm{~mm}$ in the transversal direction (i.e., tangential to the phantom cross-section).

- Furthermore, in the aim of highlighting the detection capabilities of the I-MUSIC independently from a priori information on the detection scene, apart from the calibration of the network analyzer at its port, no further calibration was carried out (i.e., no response from the tank was eliminated).
Significant research and sophisticated engineering have been dedicated in our efforts to mitigate mechanical uncertainties in the data-acquisition system for microwave breast cancer imaging. ${ }^{21,22}$

With respect to measured reconstructions showing the superiority of the I-MUSIC approach in relative terms, it would be useful to estimate how measurement uncertainties alter the achievable performance respect with an ideal controlled environment. To this end, experimental measurements taken on the oil-in-gelatin phantom were benchmarked by numerical simulations of equivalent scenarios. An equivalent 2D phantom was numerically implemented in CST Microwave Studio and scanned by the three antennas in Sec. 3. Following the very same procedure adopted for measurements, the phantom was scanned across the same 36 angles. Reconstructions obtained from numerical data are shown in Fig. 5. Although the absence of mechanical uncertainties in the numerical

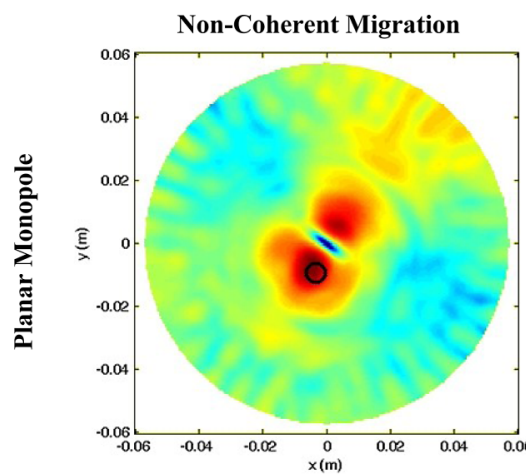

(a)

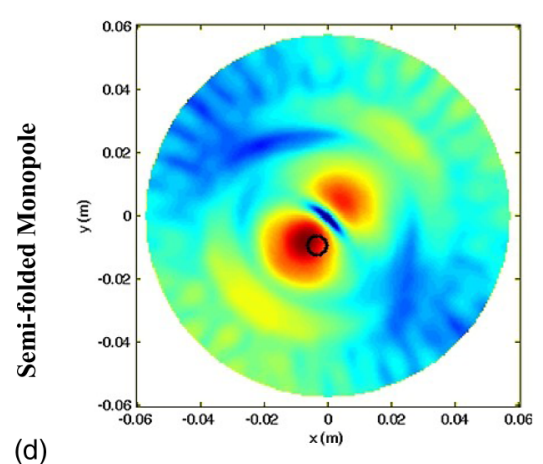

(d)

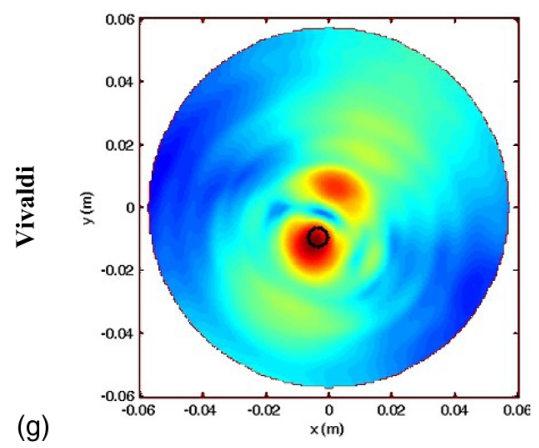

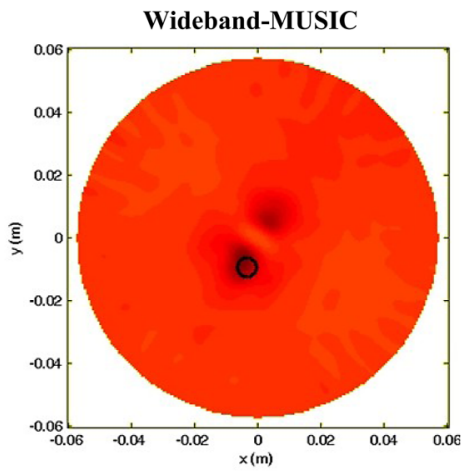

(b)

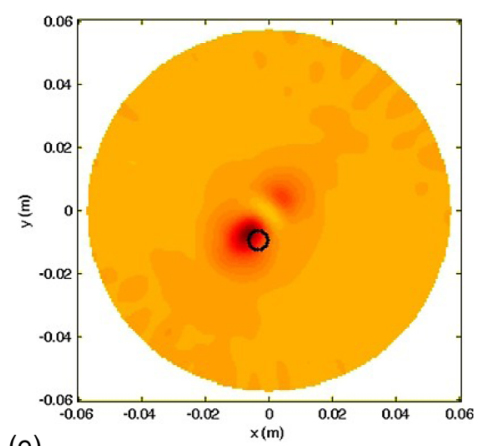

(e)

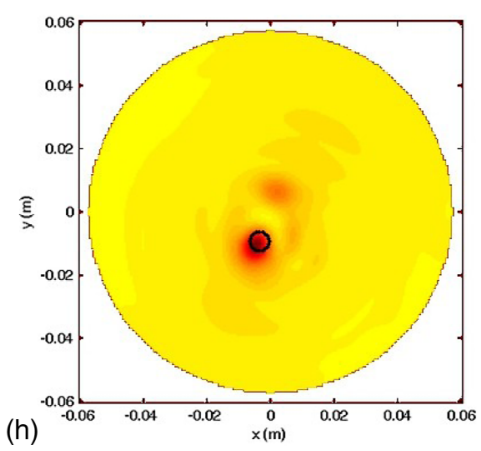

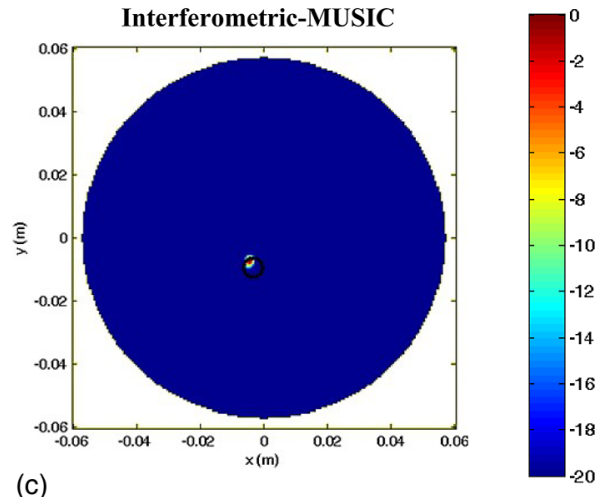

(c)

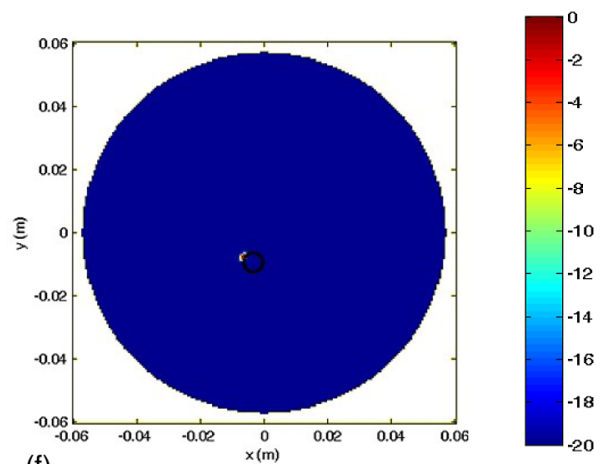

(f)

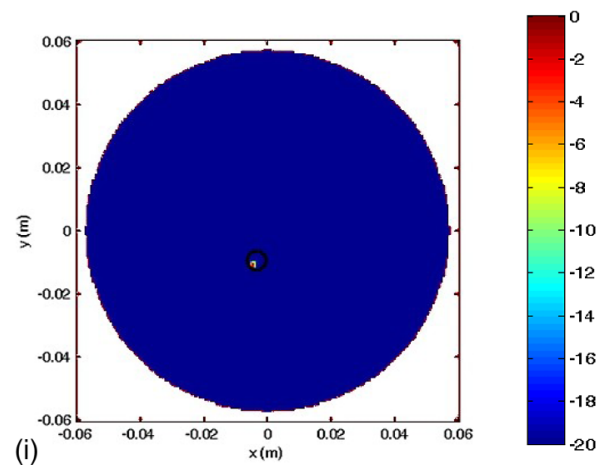

FIG. 5. Reconstructions based on numerical results. Each reconstruction is normalized to the corresponding maximum value and is expressed in decibel-scale. Tumor marked with black circle. 
TABLE II. Reconstruction metrics. Numerical results.

\begin{tabular}{lccc}
\hline \hline Method & $\begin{array}{c}\text { SCR } \\
(\mathrm{dB})\end{array}$ & $\begin{array}{c}\text { SMR } \\
(\mathrm{dB})\end{array}$ & $\begin{array}{c}\text { SD } \\
(\mathrm{mm})\end{array}$ \\
\hline Coplanar monopole & & & \\
$\quad$ N-M & 1.11 & 10.65 & 3.27 \\
$\quad$ WB-MUSIC & 1.92 & 3.81 & 2.71 \\
$\quad$ I-MUSIC & 13.52 & 81.08 & 2.71 \\
Semifolded monopole & & & \\
$\quad$ N-M & 3.12 & 12.25 & 4.16 \\
WB-MUSIC & 3.82 & 6 & 3.37 \\
$\quad$ I-MUSIC & 7.61 & 78.07 & 3.88 \\
Vivaldi & & & \\
$\quad$ N-M & 3.47 & 12.53 & 2.08 \\
$\quad$ WB-MUSIC & 4.71 & 7.30 & 1.61 \\
I-MUSIC & 10.11 & 85.02 & 1.61 \\
\hline \hline
\end{tabular}

environment leads to a certain disagreement with experimental results, simulations confirm the reconstructions obtained through measurements with clear outperforming outcomes achieved with the I-MUSIC and the Vivaldi antenna. SCR, SMR, and SD metrics were also calculated for the numerical case and figures are summarized in Table II.

\section{DISCUSSION}

The fundamental problem addressed in this study is how to reduce the dependence from a priori characterization of the antenna response for microwave breast cancer radar detection. With the antenna placed in a near-field scenario and coupling with breast tissues which are differently distributed from patient to patient, the definition of its response is strongly limited. Previous attempts based on a priori information extracted from measurements or numerical simulations show partial efficacy that results in inaccurate diagnostics. By setting a complete experimental and numerical assessment carried out with antennas with different coupling mechanisms, the I-MUSIC approach maintains its outperforming trend against otherwise limited detection achieved with migration and WB-MUSIC. Considering that preliminary antenna response characterization and equalization were never exploited for the detection and image reconstructions, I-MUSIC can reduce the requirement for a priori antenna information. Only two assumptions were made in this study: the first one is relative to the clutter-rejection procedure by discarding the contribution corresponding to the highest singular value; the second one regards the choice of an equivalent breast permittivity $\varepsilon_{r \text {-equivalent }}$ equal to $\varepsilon_{r \text {-coupling_medium }}$ for the formation of the reference Green's function. Both assumptions were extensively justified in Sec. 4.

In order to highlight the advantages of the I-MUSIC, a comparison was made with two linear noncoherent methods (i.e., wideband MUSIC and noncoherent migration) that are commonly used in the literature. With both experimental measurements and numerical benchmark, the I-MUSIC shows higher performance due to its reduced dependence from a priori, and often unfeasible, characterization. The delocalization of the detected target from the actual position is visible in Fig. 4 and it is quantified in Table I in terms of SD. This affects all detection methods under comparison in both experimental and numerical cases, although it is emphasized in measured data sets due to mechanical uncertainties. This is mainly due to the mismatch between the adopted equivalent breast permittivity in the reference Green's function and the actual unknown permittivity mapping of the detection scene. The dependence of this delocalization effect from such mismatch was analyzed by Solimene et al. ${ }^{24}$ in controlled canonical scenarios and it draws the attention toward further developments of the I-MUSIC approach.

\section{CONCLUSIONS}

A 2D oil-in-gelatin breast phantom was made for the evaluation of the scanning capability of the I-MUSIC technique using three antennas with different radiation/coupling properties. The I-MUSIC was also compared to noncoherent migration and WB-MUSIC approaches. Measurements were taken and equivalent numerical simulations were carried out by scanning the phantom in a multi-monostatic configuration across 36 uniformly distributed angles. Apart from the calibration of the VNA at the antenna terminals, no further characterization of the detection scene was carried out. The response of the tank was not subtracted. Neither was a response from an equivalent tumorless phantom considered. Reconstructions were obtained from datasets without any preprocessing procedure apart from the TSVD clutter-rejection technique described in Sec. 4 [Eq. (1)]. The I-MUSIC method offers better focusing capabilities and larger dynamic range between clutter and tumor levels when compared to the other algorithms under consideration. In particular, the antipodal Vivaldi antenna outperforms the coplanar and the semifolded monopoles as it enables more pronounced artifact clutter mitigation in reconstructions. Considering the limited 1.5:1 dielectric contrast between tumor and fibroconnective tissues, the 5-mm-diameter of the tumor, and the independence from a priori antenna characterization, the I-MUSIC system presents promising features for early stage breast cancer diagnostics.

\section{ACKNOWLEDGMENTS}

This work was supported by Italian Ministry of University and Research through the FIRB initiative under the project MICENEA (RBFR12A7CD), POR Campania FSE 2007/2013-“MASTRI", the COST Action IC1102 VISTA, and the COST Action TD1301 MiMed. The MRI scanning of the phantom was carried out by Professor Fagan in the Centre for Advanced Medical Imaging, St James's Hospital, Trinity College Dublin, Ireland.

\section{APPENDIX: BEAMFORMING AS A PARTICULAR CASE OF NONCOHERENT MIGRATION}

In this section the relationship between the noncoherent migration and the beamforming algorithm is briefly clarified. 
Let us start by the recalling the beamforming equation

$$
\Phi_{\mathrm{BF}}\left(r_{k}\right)=\int_{T}^{T+T_{w}}\left[\sum_{m} s_{\mathrm{dm}}\left(t-T+\tau_{m}\left(r_{k}\right)\right]^{2} d t\right.
$$

where $s_{\mathrm{dm}}(\cdot)$ is the signal collected at $r_{0 m}, T=\max \left\{\tau_{m}\right\}$ (over the trial positions and the sensors positions), $\tau_{m}\left(r_{k}\right)$ $=2\left|r_{0 m}-r_{k}\right|^{2} / v$ ( $v$ being the assumed propagation speed) and $W=\left[T, T+T_{W}\right]$ is the time integration window. In practical situations time $T$ should also account for the time delay due to the propagation inside the antenna structure. By accepting that no information on the antenna is available, choice of $W$ is not immediate. Here, time windowing is completely discarded so that Eq. (A1) becomes

$$
\Phi_{\mathrm{BF}}\left(r_{k}\right)=\int\left[\sum_{m} s_{\mathrm{dm}}\left(t-T+\tau_{m}\left(r_{k}\right)\right]^{2} d t\right.
$$

which by Parseval's formula can be rewritten as

$$
\Phi_{\mathrm{BF}}\left(r_{k}\right)=\int_{B}\left|\sum_{m} S_{\mathrm{dm}}(f) \exp \left[j 2 \pi f \tau_{m}\left(r_{k}\right)\right]\right|^{2} d f,
$$

where $B$ is the signals frequency band and $S_{\mathrm{dm}}(f)$ denotes the Fourier transform of $s_{\mathrm{dm}}(t)$. By accounting for that, only a discrete set of frequencies is employed and by recalling the 2D Green's function asymptotic behavior, Eq. (A3) can be recast as

$$
\Phi_{\mathrm{BF}}\left(r_{k}\right)=\sum_{n_{f}=1}^{N_{f}}\left\|S_{d}^{n_{f} H} \tilde{A}^{k}\left(f_{n_{f}}\right)\right\|^{2},
$$

where $\tilde{A}^{k}\left(f_{n}\right)$ is the Green's function column as in Eq. (3) once the amplitude spreading terms has been compensated. Now, it is immediate to realize that Eqs. (A4) and (8) coincide apart from some normalizing weights defined in Eq. (5).

\footnotetext{
a) Author to whom correspondence should be addressed. Electronic mail: giuseppe.ruvio@dit.ie

1"Breast cancer: prevention and control," World Health Organization, http:// www.who.int/cancer/detection/breastcancer/en/.

${ }^{2}$ P. T. Huynh, A. M. Jorolimek, and S. Dave, "The false-negative mammogram," RadioGraphics 18(5), 1137-1154 (1988).

${ }^{3}$ L. E. Larsen and J. H. Jacobi, "Microwave interrogation of dielectric targets. Part I. By scattering parameters," Med. Phys. 5, 500-508 (1978).

${ }^{4}$ N. K. Nikolova, "Microwave imaging for breast cancer," IEEE Microwave Mag. 17(7), 78-94 (2011).

${ }^{5}$ M. Lazebnik, L. McCartney, D. Popovic, C. B. Watkins, M. J. Lindstrom, J. Harter, S. Sewall, A. Magliocco, J. H. Booske, M. Okoniewski, and S. C. Hagness, "A large-scale study of the ultrawideband microwave dielectric properties of normal breast tissue obtained from reduction surgeries," Phys Med. Biol. 52, 2637-2656 (2007).

${ }^{6}$ M. Lazebnik, D. Popovic, L. McCartney, C. B. Watkins, M. J. Lindstrom, J. Harter, S. Sewall, T. Ogilvie, A. Magliocco, T. M. Breslin, W. Temple, D. Mew, J. H. Booske, M. Okoniewski, and S. C. Hagness, "A large-scale study of the ultrawideband microwave dielectric properties of normal, benign and malignant breast tissues obtained from cancer surgeries," Phys. Med. Biol. 52, 6093-6015 (2007).

${ }^{7}$ S. Y. Semenov, A. E. Bulyshev, A. Abubakar, V. G. Posukh, Y. E. Sizov, A. E. Souvorov, P. M. van den Berg, and T. C. Williams, "Microwavetomographic imaging of the high dielectric-contrst objects using different
}

image-reconstruction approaches," IEEE Trans. Microwave Theory Tech. 25(7), 2284-2294 (2005).

${ }^{8}$ R. Scapaticci, I. Catapano, and L. Crocco, "Wavelet-based adaptive multiresolution inversion for quantitative microwave imaging of breast tissues," IEEE Trans. Antennas Propag. 60(8), 3217-3726 (2012).

${ }^{9}$ M. Donelli, I. Craddock, D. Gibbins, and M. Sarafianou, "A three-dimensional time domain microwave imaging method for breast cancer detection based on an evolutionary algorithm," Prog. Electromagn. Res. M 18, 179-195 (2011).

${ }^{10}$ T. Isernia, V. Pascazio, and R. Pierri, "On the local minima in a tomographic imaging technique,” IEEE Trans. Geosci. Rem. Sens. 39, 1596-1607 (2001).

${ }^{11}$ M. Guardiola, S. Capdevila, J. Romeu, and L. Jofre, "3-D microwave magnitude combined tomography for breast cancer detection using realistic breast models," IEEE Antenn. Wireless Propag. Lett. 11, 1622-1625 (2012).

${ }^{12}$ R. C. Conceicao, M. O'Halloran, E. Jones, and M. Glavin, "Investigation of classifiers for early-stage breast cancer based on radar target signatures," Prog. Electromagn. Res. 105, 295-311 (2010).

${ }^{13}$ H. M. Jafari, M. J. Deen, S. Hranilovic, and N. K. Nikolova, "A study of ultrawideband antennas for near-field imaging," IEEE Trans. Antennas Propag. 55(4), 1184-1188 (2007).

${ }^{14}$ D. Flores-Tapia and S. Pistorius, "Real time breast microwave radar image reconstruction using circular holography: A study of experimental feasibility," Med. Phys. 38, 5420-5431 (2011).

${ }^{15}$ D. Gibbins, M. Klemm, I. J. Craddock, J. A. Leendertz, A. Preece, and R. Benjamin, "A comparison of a wide-slot and a stack-patch antenna for the purpose of breast cancer detection," IEEE Trans. Antennas Propag. 58(3), 665-674 (2010)

${ }^{16}$ X. Li, S. C. Hagness, M. K. Choi, and D. W. Van der Weide, "Numerical and experimental investigation of an ultrawideband ridged pyramidal horn antenna with curved launching plane for pulse radiation," IEEE Antenn. Wireless Propag. Lett. 2, 259-262 (2003).

${ }^{17}$ G. Ruvio, R. Solimene, A. D'Alterio, M. J. Ammann, and R. Pierri, "RF breast cancer detection employing a non-characterized vivaldi antenna and a MUSIC-like algorithm," Int. J. RF Microwave Comput.-Aided Eng. 23(5), 598-609 (2013).

${ }^{18}$ J. Bourqui, E. Fear, and M. Okoniewski, "Balanced antipodal Vivaldi antenna with dielectric director for near-field microwave imaging," IEEE Trans. Antennas Propag. 58, 2318-2326 (2010).

${ }^{19}$ E. Porter, E. Kirshin, A. Santorelli, M. Coates, and M. Popovic, "Timedomain multistatic radar system for microwave breast screening," IEEE Antenn. Wireless Propag. Lett. 12, 229-232 (2013).

${ }^{20}$ P. M. Meaney, M. W. Fanning, T. Reynolds, C. J. Fox, Q. Fang, S. P. Poplack, and K. D. Paulsen, "Initial clinical experience with microwave breast imaging in women with normal mammography," Acad. Radiol. 14, 207-218 (2007).

${ }^{21}$ E. Fear, J. Bourqui, C. Curtis, D. Mew, B. Docktor, and C. Romano, "Microwave breast imaging with a monostatic radar-based system: A study of application to patients," IEEE Trans. Microwave Theory Tech. 61(5), 21192128 (2013).

${ }^{22}$ T. Henriksson, M. Klemm, D. Gibbins, J. Leendertz, T. Horseman, A. W. Preece, R. Benjamin, and I. J. Craddock, "Clinical trials of a multistatic UWB radar for breast imaging," in IEEE Antennas and Propagation Conference (LAPC), Loughborough, UK, 2011.

${ }^{23}$ M. D. Hossain, A. S. Mohan, and M. J. Abedin, "Beamspace time-reversal microwave imaging for breast cancer detection," IEEE Antennas Wireless Propag. Lett. 12, 241-244 (2013).

${ }^{24}$ R. Solimene, G. Ruvio, A. Dell'Aversano, A. Cuccaro, M. J. Ammann, and R. Pierri, "Detecting point-like sources of unknown frequency spectra," Prog. Electromagn. Res. B 50, 347-364 (2013).

${ }^{25} \mathrm{G}$. Ruvio, R. Solimene, A. Cuccaro, and M. J. Ammann, "Comparison of non-coherent linear detection algorithms applied to a 2-D numerical breast model," IEEE Antenn. Wireless Propag. Lett. 12, 853-856 (2013).

${ }^{26}$ E. Zastrow, S. K. Davis, M. Lazebnik, F. Kelcz, B. D. Van Veen, and S. C. Hagness, "Database of 3D grid-based numerical breast phantoms for use in computational electromagnetics simulations," http://uwcem.ece.wisc.edu/ MRIdatabase/InstructionManual.pdf.

${ }^{27}$ E. J. Bond, X. Li, S. C. Hagness, and B. D. Van Veen, "Microwave imaging via space-time beamforming for early detection of breast cancer," IEEE Trans. Antennas Propag. 51(8), 1690-1705 (2003).

${ }^{28}$ M. Klemm, J. A. Leendertz, D. Gibbins, I. J. Craddock, A. Preece, and R. Benjamin, "Microwave radar-based differential breast cancer imaging: Imaging in homogeneous breast phantoms and low contrast scenarios," IEEE Trans. Antennas Propag. 57(7), 2337-2344 (2010). 
${ }^{29} \mathrm{~W}$. Zhi and F. Chin, "Entropy-based time window for artifact removal in UWB imaging of breast cancer detection," IEEE Signal Process. Lett. 13(10), 585-588 (2006).

${ }^{30}$ M. Sarafianou, I. Craddock, and T. Henriksson, "Towards enhancing skin reflection removal and image focusing using a 3-D breast surface reconstruction algorithm," IEEE Trans. Antennas Propag. 61(10), 5343-5346 (2013).

${ }^{31}$ A. Mashal, F. Gao, and S. C. Hagness, "Heterogeneous anthropomorphic phantoms with realistic dielectric properties for microwave breast imaging experiments," Microwave Opt. Technol. Lett. 53(8), 1896-1902 (2011).

${ }^{32}$ M. Lazebnik, E. L. Madsen, G. R. Frank, and S. C. Hagness, "Tissuemimicking phantom materials for narrowband and ultrawideband microwave applications," Phys. Med. Biol. 50, 4245-4258 (2005).

${ }^{33}$ F. Ahmad and M. G. Amin, "Non-coherent approach to through-the-wall radar localization,” IEEE Trans. Aerosp. Electron. Syst. 42(4), 1405-1419 (2006).

${ }^{34}$ M. E. Yavuz and F. L. Teixeira, "On the sensitivity of time-reversal imaging techniques to model perturbations," IEEE Trans. Antennas Propag. 56, 834-843 (2008).

${ }^{35}$ M. O'Halloran, E. Jones, and M. Glavin, "Beamforming for the early detection of breast cancer," IEEE Trans. Biomed. Eng. 57(4), 830-840 (2010).
${ }^{36}$ X. Zeng, A. Fhager, M. Persson, P. Linner, and H. Zirath, "Accuracy evaluation of ultrawideband time domain systems for microwave imaging," IEEE Trans. Antennas Propag. 59(11), 4279-4285 (2011).

${ }^{37}$ P. M. Meaney, A. H. Golnabi, N. R. Epstein, S. D. Geimer, M. W. Fanning, and J. B. Weaver, "Integration of microwave tomography with magnetic resonance for improved breast imaging," Med. Phys. 40, 103101 (13pp.) (2013).

${ }^{38}$ Keith D. Paulsen, "Breast lesion classification using ultrawideband early time breast lesion response," IEEE Trans. Antennas Propag. 58(8), 2604-2613 (2010).

${ }^{39}$ G. Ruvio, R. Solimene, A. Cuccaro, J. E. Browne, D. Gaetano, and M. J. Ammann, "Experimental microwave breast cancer detection with oil-in gelatin phantom," in IEEE International Conference Electromagnetics in Advanced Applications (ICEAA), Torino, Italy, 2013.

${ }^{40}$ G. Ruvio, R. Solimene, and M. J. Ammann, "Evaluation of antenna types for rf breast cancer imaging using 2-layer planar tissue model,' in 2010 European Microwave Conference (EuMC), Paris, France, 28-30 September (IEEE, New York, NY, 2010), pp. 212-215.

${ }^{41}$ M. A. Elahi, M. Glavin, E. Jones, and M. O'Halloran, "Artifact removal algorithms for microwave imaging of the breast," Prog. Electromagn. Res. 141, 185-200 (2013). 